\title{
Photometric estimates of stellar masses in high-redshift galaxies
}

\author{
S. Berta ${ }^{1}$, J. Fritz ${ }^{1}$, A. Franceschini ${ }^{1}$, A. Bressan ${ }^{2}$, and C. Lonsdale ${ }^{3}$ \\ ${ }^{1}$ Dipartimento di Astronomia, vicolo dell'Osservatorio 2, 35122 Padova, Italy \\ 2 Osservatorio Astronomico di Padova, vicolo dell'Osservatorio 5, 35122 Padova, Italy \\ ${ }^{3}$ Infrared Processing \& Analysis Center, California Institute of Technology 100-22, Pasadena, CA 91125, USA
}

Received 4 September 2003 / Accepted 3 February 2004

\begin{abstract}
We present a new tool for the photometric estimate of stellar masses in distant galaxies. The observed source spectral energy distributions are fitted by combining sets of various simple stellar populations, with different normalizations and different amounts of dust extinction, for a given (Salpeter) IMF. This treatment gives us the best flexibility and robustness when dealing with the widest variety of physical situation for the target galaxies, including inactive spheroidal and active starburst systems. We tested the code on three classes of sources: complete samples of dusty ISO-selected starbursts and of $K$-band selected ellipticals and S0s in the HDF South, and a representative sample of $z \sim 2$ to 3 Lyman-break galaxies in the HDF North. We pay particular attention in evaluating the uncertainties in the stellar mass estimate, due to degeneracies in the physical parameters, different star formation histories and different metallicities. Based on optical-NIR photometric data, the stellar masses are found to have overall uncertainties of a factor of $\sim 2$ for E/S0s, while for the starburst population these rise to factors $2-5$ (even including ISO/15 $\mu \mathrm{m}$ photometric data), and up to $\geq 10$ for Ly-break galaxies. Our analysis reveals in any case the latter to correspond to a galaxy population significantly less massive $\left(M<\right.$ a few $\left.10^{10} M_{\odot}\right)$ than those observed at lower redshifts (for which typically $M>$ several $10^{10} M_{\odot}$ ), possibly indicating substantial stellar build-up happening at $z \sim 1$ to 2 in the field galaxy population. Using simulated deep SIRTF/IRAC observations of starbursts and Lyman-break galaxies, we investigate how an extension of the wavelength dynamic range will decrease the uncertainties in the stellar mass estimate, and find that they will reduce for both classes to factors of 2-3, comparable to what found for E/S0s and good enough for statistically reliable determinations of the galaxy evolutionary mass functions.
\end{abstract}

Key words. galaxies: active - galaxies: starburst - galaxies: fundamental parameters - galaxies: high-redshift infrared: galaxies

\section{Introduction}

One of the still open critical questions of modern cosmology is to understand the epoch at which galaxies assembled the bulk of their stellar content. In the so-called monolithic scenario, the assembly of galaxies took place on rapid timescales at high redshifts, then galaxies evolved passively to present days. On the contrary, in the hierarchical scenario (Kaufmann \& Charlot 1998) galaxy formation is predicted to be a more continuous process and elliptical galaxies to assemble through merging of lower mass disc galaxies at moderate redshifts.

Many authors have analysed the evolution of the global star formation rate (SFR) through cosmic history, back to $z \geq 3$, using different observational tracers of the SFR in distant galaxies. It is generally accepted that galaxies produced stars more actively in the past than today, but the true rates of star formation are affected by a variety of uncertainties and biases,

Send offprint requests to: S. Berta, e-mail: berta@pd. astro.it expecially related to the amount of dust in galaxies and its effect on the SFR tracers.

A complementary approach consists in measuring the dynamical or stellar masses of distant galaxies, instead of the instantaneous SFR. Dynamical masses are directly connected to theoretical predictions, but very difficult to measure, requiring high spatial and spectral resolution spectroscopy of selected samples of faint high-redshift galaxies. Stellar masses, on the contrary, are less well determined by theory, but have the advantage that can be derived using multiband optical and near-IR photometry as a powerful alternative to time-expensive spectroscopic investigations. Indeed galaxy near-IR SEDs show a moderate dependence on the age of the contributing stars (e.g. Franceschini \& Lonsdale 2003), because in a typical galaxy the stellar mass is dominated by low-mass stars, with evolutionary timescales of the order of the Hubble time. As discussed by several authors (e.g. Lancon et al. 1999; Origlia \& Oliva 2000), these moderate-mass stars emit predominantly in the near infrared (NIR, $J$-to- $K$ restframe bands), especially during 
their cool giant phase, and are only slightly affected by dust extinction.

In this work we present a new spectro-photometric synthesis tool, aimed at the estimate of stellar masses in galaxies from a thorough analysis of their spectral energy distributions (SEDs). The tool combines a set of simple stellar populations of different ages, assuming different star formation rates and different amounts of extinction for each. The code is tested by fitting the optical-NIR broadband SEDs of two samples of intermediate $(z=0.5-1.5)$ redshift galaxies, luminous IR-selected starbursts in the HDFS (Franceschini et al. 2003) and mostly passively-evolving $K$-band selected ellipticals from Rodighiero et al. (2001). A benchmark higher-redshift population, also targeted by our analysis, was extracted from a sample of $z \sim 2-3$ Lyman-break galaxies (Papovich et al. 2001).

We pay particular attention to the uncertainties in the mass estimates, due to degeneracies in extinction, age and star formation history, and discuss how forthcoming near-to-mid infrared data from SIRTF will further constrain the photometric mass estimate for these sources.

The paper is structured as follows. Section 2 describes the three samples of selected sources. Section 3 presents the model tools used to fit the observed optical-NIR spectral energy distributions of the sources and the different star formation histories assumed for the two classes of galaxies analyzed. Section 4 discusses our results, with a particular care for degeneracies. Section 5 shows perspectives for future SIRTF/IRAC observations and Sect. 6 summarizes our conclusions. We assume a $H_{0}=65\left[\mathrm{~km} \mathrm{~s}^{-1} \mathrm{Mpc}^{-1}\right], \Omega_{m}=0.3, \Omega_{\Lambda}=0.7$ cosmology.

\section{The samples}

Our analysis compares synthetic spectra of a new spectrophotometric code with broad-band observational data to estimate the galaxy's main physical parameters, particularly the total stellar mass, but also, at the same time, age, extinction and starformation rate. Our aim here is to investigate in particular the uncertainties in the stellar mass estimates from degeneracies in the solutions due to the sparse characterization of the observational spectrum.

We compare with test cases provided by populations of luminous starburst galaxies and spheroidal galaxies, the two representing somewhat limiting physical situations. In the former class, the presence of red giants and dust-reddened young stars complicates the analysis and widens the uncertainty range. On the other hand, for passively evolving spheroids the uncertainties are expected to be much lower.

A third situation explored here is higher redshift $(z \sim 2-3)$ Lyman-break galaxies, to show how the poorer coverage of the rest-frame spectral energy distribution is affecting the uncertainties in the parameters.

\subsection{Mid-IR selected starbursts}

The rich variety of high photometric quality data in the Hubble Deep Field South and its Flanking Fields allows us to build accurate SEDs for galaxies. We have selected a sample of intermediate-redshift sources detected by ISO in the
LW3 $\left(\lambda_{\mathrm{c}}=15 \mu \mathrm{m}\right)$ band (Franceschini et al. 2003; Oliver et al. 2002) to a flux limit of $S_{\mathrm{LW} 3}=90 \mu \mathrm{Jy}$. Franceschini et al. (2003) compare the ISO source list with those from the Deep ESO Imaging Survey (EIS Deep, da Costa et al. 1998) and with the optical UBVRI catalogue by Teplitz et al. (1998), and identify 35 extragalactic objects. Of these 35 sources, 3 are likely to be hosting an AGN component and for another 2 not enough photometric data are available. For the remaining 30 galaxies, UBVRIJHK photometry is available (Da Costa et al. 1998; Tepliz et al. 1998), as well as the LW3 $15 \mu \mathrm{m}$ ISO flux and - in some cases - the LW2 $6.7 \mu \mathrm{m}$ flux. For 17 sources spectroscopic redshifts are available from Rigopoulou et al. (2000) and Franceschini et al. (2003); for the 13 others we rely on the photometric estimate.

\subsection{K-band selected ellipticals and SOs}

As representative of this class we chose a morphologically selected E/S0 sample by Rodighiero et al. (2001) in the HDFS. Based on a SExtractor analysis (Bertin \& Arnouts 1996) of the EIS-Deep $K$ band SOFI image (Da Costa et al. 1998), they computed a completeness limit of $K \simeq 20.2$ (Vega magnitude). Among this list, the subpopulation of spheroidal galaxies was identified from both visual morphological inspection and using the authomatic analysis tool GASPHOT (Pignatelli \& Fasano 1999). From this, a sample of $29 \mathrm{E} / \mathrm{S} 0$ brighter than $K=20.1$, in the WFPC2 HDFS field was selected.

Among these sources, data in the whole UV-to-NIR spectral range are only available for 23 galaxies: $U B V I$ from HST (filters $F 300 W, F 450 W, F 606 W, F 814 W$, Williams et al. 1996) and JHK from EIS Deep (SOFI/NTT, Da Costa et al. 1998) photometry. For 10 of these sources spectroscopic redshifts are available from Vanzella et al. (2003) and Sawicki \& Mallen-Ornelas (2003). For the remaining, the photometric estimate is reliable to within $5 \%$.

\subsection{Lyman break galaxies}

Papovich et al. (2001) report the analysis of stellar populations of a sample of Lyman-break galaxies in the Hubble Deep Field North, selected through the $U$ dropout tecnique, up to redshift 3.4.

The publicly available photometric data consist of the original optical WFPC2 $F 303 W, F 450 W, F 606 W, F 814 W$ HDFN observations by Williams et al. (1996), the NICMOS $F 160 \mathrm{~W}$ and ground based (IRIM) Ks data by Papovich et al. (2001) and the $J$ band magnitudes reported by Sawicki \& Yee (1998), obtained with IRIM on the KPNO $4 \mathrm{~m}$ Mayall telescope. Redshifts have been spectroscopically derived by various authors (e.g. Steidel et al. 1996; Lowental et al. 1997).

We have selected a sub-sample of 8 sources between $z \sim$ 2-3: this redshift range is the one where future SIRTF/IRAC observations will be more effective in constraining the stellar masses of such objects. 


\section{The model tool}

The observed SEDs for the target galaxies span wide ranges of rest-frame wavelengths $(0.2-1.4 \mu \mathrm{m}$ at the mean redshift of the starburst sample, $z=0.6$ ), and therefore are sensitive to stellar populations over a wide range of ages and mass/luminosity ratios and to different aspects of galaxies star formation histories. In the starburst galaxy case the observed UV-optical SED is strongly affected by dust extinction. Dust re-radiation is expected in the mid- to far-IR. The relative contributions of young red stars from older stellar populations can then be constrained with ISO mid-IR observations.

\subsection{Generalities on the spectro-photometric model}

To generate the synthetic spectra, we have developed a new automatic analysis tool as an upgrade of the spectral synthesis code by Poggianti et al. (2001). We modified it on the computational side, in versatility and improved user-interface.

Each observed SED is modelled as a combination of a set of simple stellar populations (SSP) of solar metallicity and different age. Each SSP is meant to represent a formation episode of average constant star formation rate (SFR) over a suitable time period $\Delta t$. Each single population is assumed to be extinguished by dust in a uniform screen according to the standard extinction law of the diffuse medium in our Galaxy $\left(R_{V}=A_{V} / E(B-V)=3.1\right.$, Cardelli et al. 1989; see Poggianti et al. 2001 and Berta et al. 2003 for a discussion). The extinction value $E(B-V)$ is allowed to vary from one stellar population to another, and the total spectrum is built up by summing the extinguished spectral energy distributions of all generations. However, in consideration of the fact that high extinction values are found only for the young stellar populations embedded in thick molecular clouds and that disc populations are on average affected by a moderate $A_{V}(\leq 1$, e.g. Kennicutt $1992)$, we have limited the $E(B-V)$ values for the populations with ages $\geq 10^{9}$ yrs to be less than 0.30 .

All the SSP spectra have been computed with a Salpeter initial mass function (IMF) between 0.15 and $120 M_{\odot}$, adopting the Pickles (1998) spectral library extended with Kurucz (1993) atmosphere models. The composite spectra, including both photospheric stellar and nebular (line and continuum) emission, have been obtained through the ionization code CLOUDY (Ferland 1990). This procedure provides a reliable description of single stellar generations up to a wavelength of $\sim 5 \mu \mathrm{m}$ (restframe). Beyond this wavelength, dust emission becomes typically no longer negligible.

The bulk of our analysis assumes SSPs of solar metallicity. However we can also deal with synthetic spectra with different metallicities, i.e. one third and one tenth solar, $Z=0.008$ and $Z=0.002$. As it will be subsequently discussed, the estimate of the stellar mass content of a high-redshift galaxy barely depends on the detailed assumption for metallicity.

The model SED is obtained by combining a number $N_{\text {SSP }}$ of different SSPs, weighted by different values of SFR and different amounts of extinction:

$L_{\lambda, i}^{\mathrm{unext}}=L_{\lambda, i}^{\mathrm{SSP}} \cdot S F R_{i} \cdot \Delta t_{i}$
$L_{\lambda}^{\mathrm{mod}}=\sum_{i=1}^{N_{\mathrm{SSP}}}\left(L_{\lambda, i}^{\mathrm{unext}} \times 10^{-0.4 \frac{A_{\lambda}}{A_{V}} A_{V, i}}\right)$.

The sum is intended over all the $N_{\text {SSP }}$ simple stellar generations; $L_{\lambda, i}^{\text {unext }}$ and $A_{V, i}$ are respectively the unextincted spectrum weighted by $\mathrm{SFR}_{i}$ and the extinction value for the $i$ th SSP, $L_{\lambda, i}^{\mathrm{SSP}}$ is the unextinguished luminosity of the $i$ th SSP normalized to $1 M_{\odot}, \Delta t_{i}$ is the duration of the $i$ th SSP and finally $\frac{A_{\lambda}}{A_{V}}$ is the standard extinction law $\left(R_{V}=3.1\right)$.

The result is corrected for cosmological dimming by applying the $\mathrm{K}$-correction and compared to the observed SED with:

$S_{v}=\frac{L_{v}}{4 \pi d_{\mathrm{L}}^{2}} K(v, z)$

$K(v, z)=(1+z) \frac{\int_{v_{1}}^{v_{2}} L[v(1+z)] T(v) \mathrm{d} v}{\int_{v_{1}}^{v_{2}} L(v) T(v) \mathrm{d} v}$

where $d_{\mathrm{L}}$ is the luminosity distance at the given redshift (Carroll et al. 1992) and $K(v, z)$ the K-correction factor; $T(v)$ is the filter transmission function.

\subsection{Fitting the starburst galaxy SEDs}

The SEDs of the sources in our mid-IR sample have been modelled as a combination of up to 10 SSPs, whose ages have been chosen by considering the evolutionary timescales of the associated stars. We have then considered up to four young solarmetallicity stellar generations (with ages of $10^{6}, 3 \times 10^{6}, 8 \times$ $10^{6}, 10^{7} \mathrm{yr}$ ), mostly responsible for the ionizing photons producing the emission lines and for dust heating and FIR emission; up to five intermediate-age populations $\left(5 \times 10^{7}, 10^{8}\right.$, $3 \times 10^{8}, 5 \times 10^{8}, 10^{9} \mathrm{yr}$ ) with the strongest Balmer absorption lines; finally for older generations of stars we have included a population modelled with a constant star formation rate (SFR) between 2 and 12 Gyr before the observation. This latter choice is discussed in Sect. 4.2, together with the effects of varying the SSP's metal content.

In the attempt of reducing the uncertainties in the mass estimate due to the contribution of young stars to the near-IR restframe SED, we have considered the ISO $15 \mu \mathrm{m}$ flux in addition to the $U B V R I J H K$ photometric data. The dust emission spectrum longwards of $5 \mu \mathrm{m}$ (restframe) is modelled by assuming the observed M 82 IR SED and then comparing to the flux detected by ISO in the $L W 3$ band (12 to $18 \mu \mathrm{m}$ ). The M 82 IR template is adopted as a typical spectrum of luminous IR starbursts (except some exceptional ultraluminous galaxies like Arp 220). The predicted IR emission is calculated as the difference between the unextinguished and the extinguished optical spectrum, assuming that all the flux absorbed by dust is reprocessed and re-emitted longwards of $8 \mu \mathrm{m}$. The M 82 template is then rescaled in such a way that its bolometric emission between 8 and $1000 \mu \mathrm{m}$ coincides with the dust reprocessed luminosity. Finally, the properly scaled M 82 SED is k-corrected and convolved with the $L W 3$ filter reponse to compare with the observed LW3 flux.

The M 82 template was adopted to model the IR SED of the ISOCAM sources as a fairly conservative choice. 
Elbaz et al. (2002) have shown that tight nearly linear correlations between the observed mid-IR emission and the bolometric FIR luminosity of local IR galaxies hold over $\sim 4$ orders of magnitude in $L_{\mathrm{IR}}$ (between $10^{9}$ and $10^{13} L_{\odot}$ ), involving IRAS $12 \mu \mathrm{m}$ and ISO LW2, LW3 observed fluxes. M 82, with $L_{\mathrm{IR}} \simeq 3.3 \times 10^{10} L_{\odot}$, is a prototypical galaxy, representative of dusty starbursts and laying in the middle of the Elbaz et al. (2002) correlations.

Objects not following these relations, e.g. the ULIRG Arp 220, are characterized by a much more peaked FIR emission and dust self-absorption. Adopting such an extreme galaxy as IR template would produce higher bolometric infrared luminosities for a given $15 \mu \mathrm{m}$ flux, and therefore require very high - often unphysical - extinction values for those SSPs contributing to the FIR emission. The estimate of stellar mass and its uncertainty (see Sect. 4.2) is in any case not significantly affected by this choice.

The exploration of the parameter space (see more details in Sect. 3.5 below) starts with a large number (10) of stellar populations. However, after the minimization process and for each solution with acceptable $\chi^{2}$, SSPs providing less than $1 \%$ of the observed spectrum at each wavelengths (after extinction has been applied) are identified and excluded from further consideration.

The effective number of SSPs involved in the best-fit solution of each examined object is reported in Table 1. Tipically 3-4 stellar populations then contribute to the fit of the observed SEDs, which in turn means a total of 6-8 parameters (SFR and $E[B-V]$ for each population).

The optimization process involves, at least at the beginning of the exploration, a large number of populations/parameters to ensure that the most general description of galaxy spectra and the most conservative evaluation is given of the uncertainties in the physical parameters.

\subsection{Fitting the elliptical SEDs}

It is common wisdom that the properties of local ellipticals/S0s may be reproduced by one suitable Simple Stellar Population with old age. However in some cases there are hints for the presence of $a$ ) a younger population, representative of a partial renjuvenation episode occured in the recent past; $b$ ) or a significant contribution from sub-solar metallicity stars. Both effects result in a bluening of colors.

We have analysed the spheroidal galaxy population with various methods. The first approach that we have considered consisted in adopting a simple analytical form of the star formation history (SFH) in E-type galaxies. We have achieved this by combining 55 SSPs in an almost continuous age sequence in which the stellar mass formed in each generation is obtained from:

$\operatorname{SFR}(t)=\left(\frac{t_{\mathrm{F}}-t}{t_{\mathrm{F}}}\right)^{n_{1}} \times \exp \left(-\frac{t_{\mathrm{F}}-t}{\tau_{1} \cdot t_{\mathrm{F}}}\right)$

where $t_{\mathrm{F}}$ is the epoch of formation of the galaxy (i.e. when the first star shone) and varies in the range 2-12 Gyrs. We assumed a constant small amount of extinction for all populations. Relation (5) describes the evolution of SFR in an open chemical evolution model obeying the Schmidt law. The power-law term represents the early growth of SFR.

The second method, meant to reproduce case $(a)$, consisted in the same free-form approach already described for IR starbursts, but constrained in order to avoid high extinction values and high FIR emission. The latter assumes that we have imaging data of enough quality to ensure that the object under scrutiny is a bona-fide spheroidal galaxy not affected by extinction features as revealed by dust lanes, absorption bands, etc. For the typical E/S0s in our sample, best-fits are obtained with the combination of just two SSPs, one of $1.2 \times 10^{10} \mathrm{yrs}$ and the other of $10^{9}$ yrs, plus a younger SSP needed for the blue ellipticals.

Particularly to fit the case $(b)$ sub-population, we have considered a third choice: a combination of two old simple stellar populations, both of them $1.2 \times 10^{10} \mathrm{yrs}$ old, having different values of metallicity (solar $Z=0.02$ and sub-solar $Z=0.008$ ).

As a final check, we have also considered the most general case of a completely free-form approach, as that mentioned above to fit the dusty star-forming class, but with an upper limit imposed on the mid-IR flux (as might be provided by forthcoming SIRTF multiband surveys) to constrain to amount of dust re-radiation and optical extinction.

\subsection{Fitting the Ly-break galaxy SEDs}

The spectral energy distributions of the Ly-break galaxies have been fitted assuming a discrete number of stellar populations as for the IR starbursts, but since at redshift $z \sim 2.5$ the age of the universe for the assumed cosmology is roughly $2.5 \mathrm{Gyrs}$, only SSPs up to 1 or $2 \times 10^{9}$ years - depending of $z$ - have been considered.

As for the starburst case, only stellar populations contributing to the visible and IR light by more than $1 \%$ were involved in the fit. The typical number of SSPs used is $2-3$ for Lymanbreak galaxies (see Table 3 ).

\subsection{Parameter space exploration with the Adaptive Simulated Annealing (ASA)}

For each galaxy the code randomly generates a wide set of models and compares them to the observed fluxes, by minimizing the Merit Function, which tests the accuracy of the given fit:

$\chi^{2}=\sum_{i=1}^{N}\left(\frac{S_{i}^{\mathrm{obs}}-S_{i}^{\mathrm{mod}}}{\sigma_{i}^{\mathrm{obs}}}\right)^{2}$,

where $S_{i}^{\text {obs }}$ and $S_{i}^{\text {mod }}$ are the observed and synthetic fluxes in the $i$ th photometric band, $\sigma_{i}^{\text {obs }}$ is the uncertainty on the observed flux and $N$ the number of constraints.

In the most general case there will not exist a unique minimum of the Merit Function, but rather a large number of relative minima. The exploration of the $N$-dimensional parameter space has been performed adopting the Adaptive Simulated Annealing algorithm (ASA, Ingber 1989, 2001), which is very well suited to search for a solution as close as possible to the absolute minimum, avoiding local minima. 
Table 1. Results of the stellar-mass estimate based of SEDs fitting. For each ISO/LW3 HDFS source we report redshift (Franceschini et al. 2003; photometric if within parenthesis, spectroscopic otherwise), total number of models produced by the spectrophotometric synthesis code (without the SIRTF constraints), effective number of SSPs contributing to best fit (for the optical+LW3 data, but without the SIRTF constraints), optical $\chi^{2}$ and mass of the best fit solution. The last three columns report three different estimates of the mass uncertainties due to degeneracies: $\Delta M$ at $68.3 \%$ and $95.5 \%$ confidence levels (corresponding to $1 \sigma$ and $2 \sigma$ ) and $2 \sigma$ mass range obtained simulating SIRTF/IRAC observations (only for some randomly selected sources).

\begin{tabular}{|c|c|c|c|c|c|c|c|c|}
\hline $\begin{array}{l}\text { Obj } \\
\#\end{array}$ & $z$ & $\begin{array}{l}N_{\text {tot }} \\
\text { mdls. }\end{array}$ & $\begin{array}{c}N_{\text {SSP }} \\
\text { b.f. }\end{array}$ & $\begin{array}{l}\text { Mass (b.f.) } \\
{\left[10^{11} M_{\odot}\right]}\end{array}$ & $\begin{array}{l}\chi_{\mathrm{opt}}^{2} \\
\text { b.f. }\end{array}$ & $\begin{array}{c}\Delta M_{1 \sigma} \\
{\left[10^{11} M_{\odot}\right]}\end{array}$ & $\begin{array}{c}\Delta M_{2 \sigma} \\
{\left[10^{11} M_{\odot}\right]}\end{array}$ & $\begin{array}{c}\Delta M_{\mathrm{SIRTF}} \\
{\left[10^{11} M_{\odot}\right]}\end{array}$ \\
\hline S14 & 0.41 & 420014 & 3 & 0.32 & 4.15 & $0.21-0.35$ & $0.21-0.38$ & \\
\hline S15 & $(0.55)$ & 451212 & 2 & 3.10 & 13.55 & $2.97-3.29$ & $2.61-3.49$ & \\
\hline S16 & 0.62 & 430819 & 4 & 0.29 & 4.73 & $0.23-0.28$ & $0.22-0.31$ & \\
\hline S18 & $(0.55)$ & 202806 & 4 & 5.20 & 14.37 & $5.13-5.47$ & $4.62-5.67$ & \\
\hline S20 & 0.39 & 416408 & 4 & 0.80 & 3.65 & $0.57-1.20$ & $0.57-1.26$ & \\
\hline S23 & 0.46 & 409213 & 4 & 0.97 & 8.16 & $0.45-1.16$ & $0.41-1.64$ & $0.60-1.20$ \\
\hline S25 & 0.58 & 430807 & 2 & 0.80 & 2.70 & $0.54-1.26$ & $0.44-1.47$ & $0.40-1.34$ \\
\hline S27 & 0.58 & 507625 & 4 & 4.70 & 4.56 & $4.44-5.62$ & $3.71-5.68$ & $4.17-5.54$ \\
\hline S28 & 0.58 & 476432 & 4 & 0.40 & 4.45 & $0.29-0.55$ & $0.27-0.69$ & \\
\hline S30 & $(0.40)$ & 206401 & 3 & 0.01 & 3.88 & $0.005-0.007$ & $0.005-0.025$ & \\
\hline S36 & $(0.65)$ & 198044 & 4 & 0.40 & 0.93 & $0.23-0.60$ & $0.21-0.65$ & \\
\hline S40 & 1.27 & 385289 & 4 & 1.20 & 8.71 & $1.43-2.01$ & $0.89-3.05$ & $0.80-2.00$ \\
\hline S41 & $(0.30)$ & 208838 & 3 & 0.055 & 6.43 & $0.051-0.059$ & $0.045-0.069$ & \\
\hline S43 & 0.95 & 409212 & 4 & 0.50 & 0.77 & $0.25-0.87$ & $0.22-0.94$ & $0.21-0.77$ \\
\hline S45 & $(0.65)$ & 526817 & 4 & 0.80 & 5.67 & $0.53-1.69$ & $0.24-1.71$ & $0.30-1.25$ \\
\hline S48 & $(0.30)$ & 210015 & 3 & 0.10 & 4.08 & $0.14-0.21$ & $0.058-0.21$ & \\
\hline S52 & $(0.60)$ & 278402 & 4 & 0.13 & 3.81 & $0.13-0.17$ & $0.12-0.17$ & \\
\hline S53 & 0.58 & 506477 & 3 & 1.20 & 3.34 & $0.99-1.38$ & $0.99-1.52$ & $1.20-1.38$ \\
\hline S55 & 0.76 & 477664 & 5 & 1.30 & 1.16 & $0.87-1.69$ & $0.55-1.69$ & $1.15-1.90$ \\
\hline S60 & 1.23 & 492090 & 4 & 2.50 & 14.38 & $1.91-2.89$ & $1.75-6.23$ & $2.00-3.20$ \\
\hline S62 & 0.73 & 462014 & 4 & 0.66 & 3.04 & $0.69-0.88$ & $0.66-0.95$ & \\
\hline S67 & $(1.00)$ & 424812 & 4 & 0.34 & 12.12 & $0.21-0.87$ & $0.21-0.91$ & \\
\hline S71 & $(0.45)$ & 230406 & 4 & 0.04 & 5.02 & $0.016-0.044$ & $0.013-0.067$ & \\
\hline S72 & 0.55 & 421227 & 3 & 1.40 & 12.39 & $1.14-1.30$ & $1.12-2.01$ & \\
\hline S73 & 0.17 & 441608 & 4 & 1.50 & 10.00 & $0.95-1.01$ & $0.84-1.94$ & $0.96-1.82$ \\
\hline S75 & $(0.45)$ & 222010 & 4 & 1.30 & 4.19 & $1.16-1.36$ & $0.83-1.40$ & \\
\hline S77 & $(0.40)$ & 210033 & 4 & 0.80 & 4.73 & $0.91-1.10$ & $0.47-1.16$ & $0.45-1.14$ \\
\hline S79 & 0.74 & 499221 & 3 & 0.60 & 1.60 & $0.65-1.18$ & $0.30-1.21$ & \\
\hline S82 & 0.69 & 438044 & 4 & 0.50 & 9.61 & $0.49-0.62$ & $0.37-0.83$ & $0.51-0.69$ \\
\hline S85 & $(0.40)$ & 414053 & 3 & 5.70 & 25.77 & $5.51-5.79$ & $4.78-6.19$ & \\
\hline
\end{tabular}

The heart of the Simulated Annealing method (Ingber et al. $1989,2001)$ is the thermodynamic analogy with cooling metals: at high temperatures each molecule moves with respect to the others, but when $T$ slowly decreases its mobility is gradually lost and matter reaches a pure crystalline configuration. If the cooling process is slow enough, metals can spontaneously reach this minimum energy state.

The function to be optimized can be considered similar to the total energy of the system. The Boltzmann probability distribution

$P_{E} \sim \mathrm{e}^{-\frac{E}{K T}}$ shows that even at thermal equilibrium - with temperature $T$ - a non-zero probability to find the system in an energy state higher than $K T$ does exist. Consequently it is possible for the system to move from the local energetic minimum to an excited state and subsequently evolve to an energy smaller than at start.

Following this analogy, the optimization process starts from a given state in the $N$-dimentional parameter space and then evolves from $E_{1}$ to $E_{2}$; the probability of this transition is $P_{12}=$ $\mathrm{e}^{-\frac{E_{2}-E_{1}}{K T}}$. If $E_{1}>E_{2}$, then $P>1$ : the system always moves towards lower energy states, even if a non-zero probability to increase its energy does exist.

On the other hand, small values of the temperature and large 

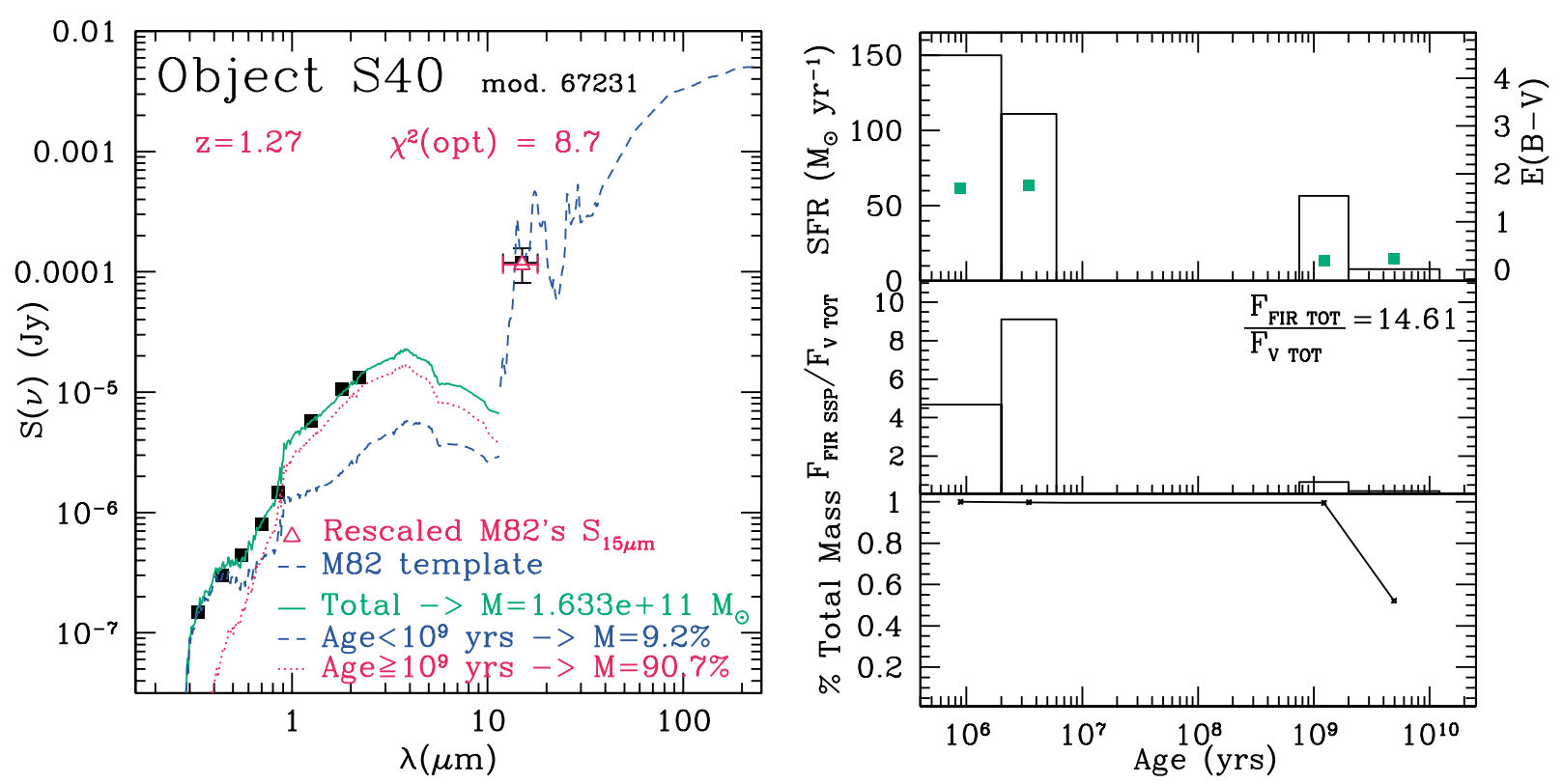

Fig. 1. HDFS/LW3 source S40 at $z=1.27$ : best fit mass is $M=1.63 e 11 M_{\odot}$. Left Panel: comparison of the observed SEDs to the best fits. The solid line is the sum of all the used SSPs; the dashed line is the sum of all the young SSPs (age $<10^{9}$ yrs); the dotted line represents the old populations (age $\geq 10^{9} \mathrm{yrs}$ ); the dashed thick line longward $5 \mu \mathrm{m}$ (restframe) is the $\mathrm{k}$-corrected rescaled $\mathrm{M} 82$ template; the square black dots are the observed photometric datapoints; the triangular open dot at $\lambda \sim 15 \mu \mathrm{m}$ is the synthetic LW3 flux (overlapping to the observed LW3 when the FIR constraint is satisfied). Right Panel top: values of SFR (histogram, left $y$-scale) and $E(B-V)$ (dots, right $y$-scale) for each simple stellar population. Middle: ratio between the FIR and $V$-band fluxes for the different SSPs. This quantity is used to asses both the contribution of various stellar populations to the FIR luminosity and the effect of extinction on each population (depressing the optical light and boosting the IR). The global ratio between the FIR and $V$ fluxes of the composite final synthetic spectrum is reported on the right. Bottom: cumulative stellar mass formed in the galaxy. The youngest stars, significantly contributing to the FIR bolometric emission, provide almost no contribution to the total luminous mass assembled in the galaxy.

energetic increments imply low probabilities for the system to evolve uphill.

In order to converge, at each $i$ th iteration, the temperature of the system is adapted according to the relation $T(i+1)=$ $R_{T} \cdot T(i)$. The adopted value for $R_{T}$ is 0.85 , as suggested by Corana et al. (1987). As $T$ declines, moves to excited energy states are suppressed and the algorithm focuses on the most promising area for optimization. On the other hand, when a new deep minimum is found, these ranges are increased, in order to avoid the system to lag on it.

Minimization ends when the $\chi^{2}$ value decreases by less than the Tolerance set by the user. Further informations on the ASA algorithm can be found at the URL http://www . ingber. com/

\section{Results}

For each of the sample sources, our simulations generate a very large number of models, each one based on a different set of physical parameters. For a typical number of computed solutions $(\sim 100000)$, the $\chi^{2}$ exploration takes between $\sim 20$ and 45 min on a $1.7 \mathrm{GHz}$ CPU PC - depending on the total number of SSPs, the adopted SFH and convergence criteria. Acceptable solutions (with reduced $\chi_{0}^{2} \sim 1$ ) are found for most of the sample galaxies. In the case of unacceptable fits, it may be that the uncertainties in the measured fluxes were underestimated, or that the distribution of the errors on parameters is not Gaussian
(Papovich et al. 2001). Finally, the SSP spectra may have intrinsic modellistic uncertainties.

\subsection{Mid-IR selected starburst galaxies}

Figure 1 illustrates the comparison of the synthetic SEDs to the observed datapoints and plots of the main physical parameters, for a representative galaxy. The left panel in the figure shows the best-fits to the SEDs. The solid line is the sum of contributions from all SSPs; the dashed line is the sum of all the young SSPs (age $<10^{9} \mathrm{yrs}$ ), while the dotted line represents the contribution of older populations (age $\geq 10^{9} \mathrm{yrs}$ ); the dashed thick line longwards of $5 \mu \mathrm{m}$ is the redshifted M 82 template; the square filled dots are the observed photometric datapoints, the open triangle at $\lambda \sim 15 \mu \mathrm{m}$ is the fit to the ISO LW3 datapoint. The identification number of the model (for the given source) and the $\chi^{2}$ value based on the optical/NIR fit only (LW3 datapoint excluded) are also reported in the plots.

The right panels of Fig. 1 show - for the various SSPs making up the best-fit model, whose age intervals are indicated in the $x$-axis - trends of the SFR (top histograms, $y$-scale on the left end), $E(B-V)$ (top panel, filled squares, $y$-scale on the right end), the ratio of the FIR flux contributed by the SSP to the total flux in the $V$ band ( $F_{\mathrm{FIR}, \mathrm{SSP}} / F_{\mathrm{V}}$, TOT, middle panel), and the cumulative distribution of the stellar mass as a function of SSP's age (bottom panel). The graph in the middle panel, in particular, details the contribution of each SSPs to the FIR bolometric 

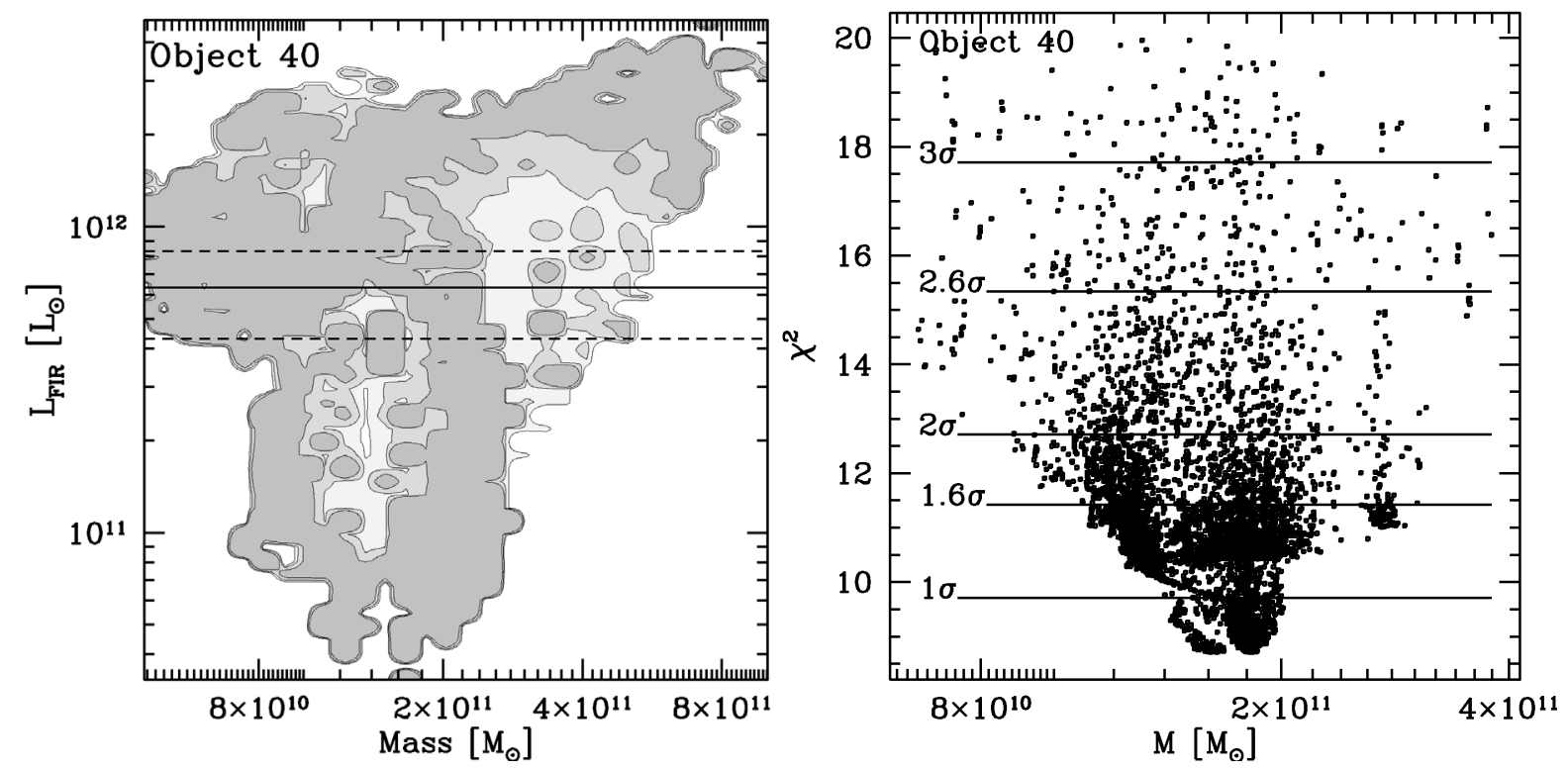

Fig. 2. Left: $\chi^{2}$ contours on the $M$ vs. $L_{\mathrm{FIR}}$ diagram, for source S40. Contours are drawn at 1, 2, 3 $\sigma$ levels, corresponding to $68.3 \%$, $95.5 \%$ and $99.7 \%$ confidence (decreasing as shade darkness increases). The solid and dashed horizontal lines represent the far-IR luminosity and uncertainty as derived from the observed LW3 flux, based on fitting with the M 82 template. Right: $\chi^{2}$ values as a function of the stellar mass of the models, for those solutions reproducing the LW3 flux within $1 \%$. The mass uncertanty range is $[0.70,3.80] \times 10^{11} M_{\odot}$.

emission of the galaxy, taking into account the effect of dust extinction in the optical light. The bottom plot shows that, typically, the youngest stars contribute to the bulk of the FIR bolometric emission of these sources, but provide almost no contribution to the optically luminous mass in the galaxy. Results for additional dusty starbursts in Hubble Deep Field South may be found in Franceschini et al. (2003).

\subsection{Degeneracies in the mass estimates for IR-selected dusty starbursts}

The left panel of Fig. 2 shows plots of $\chi^{2}$ contours as a function of stellar mass $M$ and FIR luminosity $L_{\mathrm{FIR}}$, based on fits to the optical/NIR data only (UBVRIJHK photometry). The $\chi^{2}$ contours correspond to $1,2,3 \sigma$ uncertainties. Note that even the darker $1 \sigma$ contours are extended, showing substantial degeneracies in the solutions.

The horizontal lines in Fig. 2 (left panel) mark the measured FIR bolometric $(8-1000 \mu \mathrm{m})$ luminosity and its uncertainty based on the fits to the observed LW3 flux. In only a fraction of the high- $z$ galaxies the FIR constraint is effective in reducing the uncertainties in the stellar mass: this happens in particular for the least active galaxies (those with the lowest mid-IR excess and SFR compared with the optical SED). Source S27 $(z=0.58)$ is one such case: imposing the LW3 flux constraint, the stellar mass range is reduced by more than a factor of two.

On the other hand S40, an ultraluminous galaxy at $z=1.27$, is a typical case for which the FIR information is barely useful to constrain the mass. This fact is due to the presence of highly obscured young stellar populations in the most active starbursts, which give a significant contribution to the NIR and FIR luminosity, but are not visible in the optical/NIR, because of their low $M / L$ ratio, and their contribution to the stellar mass budget is hard to constrain. For such complex situations, further mid-IR data from SIRTF will be critical, as discussed in Sect. 5 below.

As mentioned in Sect. 3.2, we have reproduced the contribution by old stellar populations with spectra obtained assuming constant SF between 2 and 12 Gyrs, since the single SSP spectra in this age range are all very similar and their $M / L$ ratio varies smoothly. This allows us to reduce the number of free parameters, when compared to a more detailed SFH. To test this procedure, we have run different simulations on several sources, detailing the SFH between 2 and 12 Gyrs into 5 different SSPs (2, 4, 7, 10 and 12 Gyrs old). Other simulations obtained by simply substituting the long-SF model with single 2 and 12 Gyrs SSPs have been run as well, in order to test the more extreme cases of masses assembled with the lowest and the highest $M / L$ values in the $2-12$ Gyrs range. The most massive and the lightest models obtained in this way may differ by a factor of $\sim 2$ in mass. However, this analysis showed that the uncertainty $\Delta M$ in stellar mass - due to different adopted models of the old population - is in fact completely contained within those of the global model fit. Indeed the $\Delta M$ due to the presence of young stellar populations dominates the global mass uncertainty.

Although we have based our analysis on solar-metallicity SSPs, we have performed a systematic analysis of how variations in metallicity may affect our results, taking into account that e.g. changing it from solar to one third solar would be equivalent to change the SSP age from $10^{9}$ to $12 \times 10^{9} \mathrm{yrs}$ (the age-metallicity degeneracy). In principle one would expect a corresponding significant difference in the $M / L$ ratio, hence on the estimated stellar mass. In fact our analysis has shown that 
Table 2. Results of the stellar-mass estimate for the ellipticals sample, based of SEDs fitting with solar metallicity SSPs. For each elliptical source in the HDFS (Rodighiero et al. 2001), spectroscopic (Vanzella et al. 2003; Sawicky \& Mallen-Ornelas 2003) or photometric (if within parenthesis, Rodighiero) redshifts, total number of models produced by the spectrophotometric synthesis code, best fit parameters and $\chi^{2}$ for the analytic SFH method (see text for details), mass of the best fit solution and $2 \sigma$ (95.5\% confidence) mass range due to degeneracies are reported. Third column reports a flag identifying red and blue sources. The SEDs of the red sources have been fitted with three different methods: $a$ ) analytic SFH, $b)$ two simple stellar populations $\left(10^{9}\right.$ and $1.2 \times 10^{10}$ yrs old $)$ with solar metallicity, $\left.c\right)$ two old populations $\left(1.2 \times 10^{10} \mathrm{yrs}\right)$ with different metallicities $(Z=0.02,0.008)$ - see also text and Fig. 3. The mass ranges reported for these objects include the results of all three fitting tecniques; on blue sources only the analytic SFH leads to good results.

\begin{tabular}{lcccccccccc}
\hline \hline Obj & $z$ & Color & $N_{\text {tot }}$ & $t_{\mathrm{F}}$ & $E(B-V)$ & $n_{1}$ & $\tau_{1}$ & $\chi^{2}$ & Mass (b.f.) & $\Delta M$ \\
$\#$ & & Flag & mdls. & b.f. [Gyrs $]$ & b.f. & b.f. & b.f. & b.f & {$\left[10^{11} M_{\odot}\right]$} & {$\left[10^{11} M_{\odot}\right]$} \\
\hline E1 & 0.512 & b & 18001 & 11.91 & 0.05 & 2.69 & 0.16 & 8.67 & 0.18 & $0.08-0.18$ \\
E2 & $(1.3)$ & b & 16848 & 11.66 & 0.02 & 3.01 & 0.16 & 11.95 & 0.83 & $0.47-1.07$ \\
E3 & $(1.3)$ & b & 23427 & 3.44 & 0.05 & 4.12 & 0.08 & 7.95 & 0.85 & $0.70-1.82$ \\
E4 & 0.414 & b & 11521 & 2.68 & 0.00 & 0.002 & 0.52 & 4.29 & 0.16 & $0.10-0.23$ \\
E5 & 0.673 & r & 129606 & 3.19 & 0.05 & 0.001 & 0.15 & 1.89 & 0.73 & $0.66-1.12$ \\
E6 & $(0.5)$ & r & 111765 & 9.20 & 0.06 & 0.002 & 0.15 & 6.31 & 0.90 & $0.52-1.16$ \\
E7 & 0.517 & b & 11287 & 4.63 & 0.10 & 5.17 & 0.11 & 6.71 & 0.10 & $0.08-0.14$ \\
E8 & 0.579 & r & 131529 & 6.68 & 0.05 & 2.85 & 0.09 & 5.31 & 0.52 & $0.42-0.83$ \\
E9 & 0.515 & b & 15841 & 2.00 & 0.00 & 5.25 & 0.10 & 6.68 & 0.05 & $0.045-0.059$ \\
E11 & 0.364 & r & 121526 & 7.17 & 0.04 & 2.35 & 0.06 & 9.92 & 0.10 & $0.07-0.16$ \\
E14 & $(1.15)$ & b & 11053 & 11.44 & 0.00 & 1.97 & 0.25 & 5.52 & 0.75 & $0.43-0.81$ \\
E16 & $(1.0)$ & b & 11283 & 11.46 & 0.05 & 0.04 & 0.26 & 8.36 & 3.42 & $2.29-3.99$ \\
E17 & $(1.25)$ & r & 136281 & 11.71 & 0.04 & 0.03 & 0.19 & 7.31 & 3.89 & $2.82-4.08$ \\
E18 & $(0.9)$ & r & 112240 & 11.46 & 0.04 & 0.02 & 0.19 & 4.58 & 0.73 & $0.38-0.89$ \\
E19 & $(0.15)$ & r & 121521 & 11.96 & 0.05 & 3.98 & 0.01 & 9.81 & 0.08 & $0.06-0.09$ \\
E20 & $(0.8)$ & b & 16087 & 2.00 & 0.00 & 0.001 & 0.34 & 9.76 & 0.12 & $0.08-0.18$ \\
E21 & 0.577 & r & 127454 & 11.28 & 0.05 & 0.04 & 0.29 & 10.00 & 0.98 & $0.61-1.16$ \\
E24 & $(0.4)$ & r & 134001 & 11.28 & 0.05 & 3.35 & 0.08 & 13.54 & 0.55 & $0.33-0.72$ \\
E25 & 0.511 & b & 13272 & 11.97 & 0.04 & 1.49 & 0.21 & 7.02 & 0.72 & $0.44-0.93$ \\
E26 & $(1.5)$ & b & 11729 & 5.52 & 0.00 & 3.69 & 0.09 & 22.34 & 2.80 & $1.89-4.12$ \\
E27 & $(0.85)$ & b & 11258 & 11.72 & 0.02 & 3.42 & 0.11 & 16.02 & 0.92 & $0.52-1.09$ \\
E28 & 0.564 & b & 11281 & 11.91 & 0.02 & 0.64 & 1.70 & 18.45 & 0.13 & $0.086-0.14$ \\
E29 & $(0.5)$ & r & 112123 & 14.35 & 0.05 & 2.76 & 0.01 & 4.79 & 0.18 & $0.078-0.32$ \\
\hline
\end{tabular}

a kind of compensations holds, such that changing the stellar metallicity requires a substantial modification of the SF history to keep an acceptable spectral fit. The result is that the mass estimate does not significantly depend on the assumed metal content, and the same applies for the mass uncertainty range. For star-forming galaxies the overwhelming uncertainty factor is instead the incidence of the young stellar population.

The stellar mass estimates are reported in Table 1 . We plot in the right panel of Fig. 2 the $\chi^{2}$ values as a function of mass for acceptable models, each dot representing a different solution. Columns 8 and 9 of Table 1 report the estimated uncertainties $\Delta M$ at 1 and $2 \sigma$ levels. The typical uncertainty in the mass values for these IR starbursts is a factor of 2-3, but may be as high as 5 in a few cases. Figure 6 shows the estimated masses against redshift.

\subsection{Elliptical galaxies}

Franceschini et al. (1998) and Rodighiero et al. (2001) found that populations of morphologically-selected spheroidal galaxies in the HDFs are characterized by a wide variety of integrated colors, including typical ellipticals with very red SEDs $(B-I>2.5$ for objects at $z \leq 1$ and $V-J>2.5$ for $z \geq 1$ ), but also many bluer objects. The $\mathrm{b} / \mathrm{r}$ flag in the third column of Table 2 identifies blue and red objects in our sample.

As previously mentioned, the SEDs of elliptical and So galaxies have been fit alternatively by assuming an analytic star formation history, or by means of the free-form approach, or the sub-solar metallicity model. The SEDs of the red subsample are equally well-fitted by all three, and the corresponding estimated values of the stellar masses agree very well within the relatively small uncertainties. For these sources, the mass range reported in Table 2 includes the results of the three fits, while the best fit value refers to the analytic SFH tecnique.

Figure 3 shows best fits for the three methods for source E5: all three yield equally good fits, although the analytic SFH gives the lowest $\chi^{2}$; the lower panels of Fig. 3 report the star formation histories and the cumulative mass distribution in the three cases. Table 2 summarizes our results; the estimated 

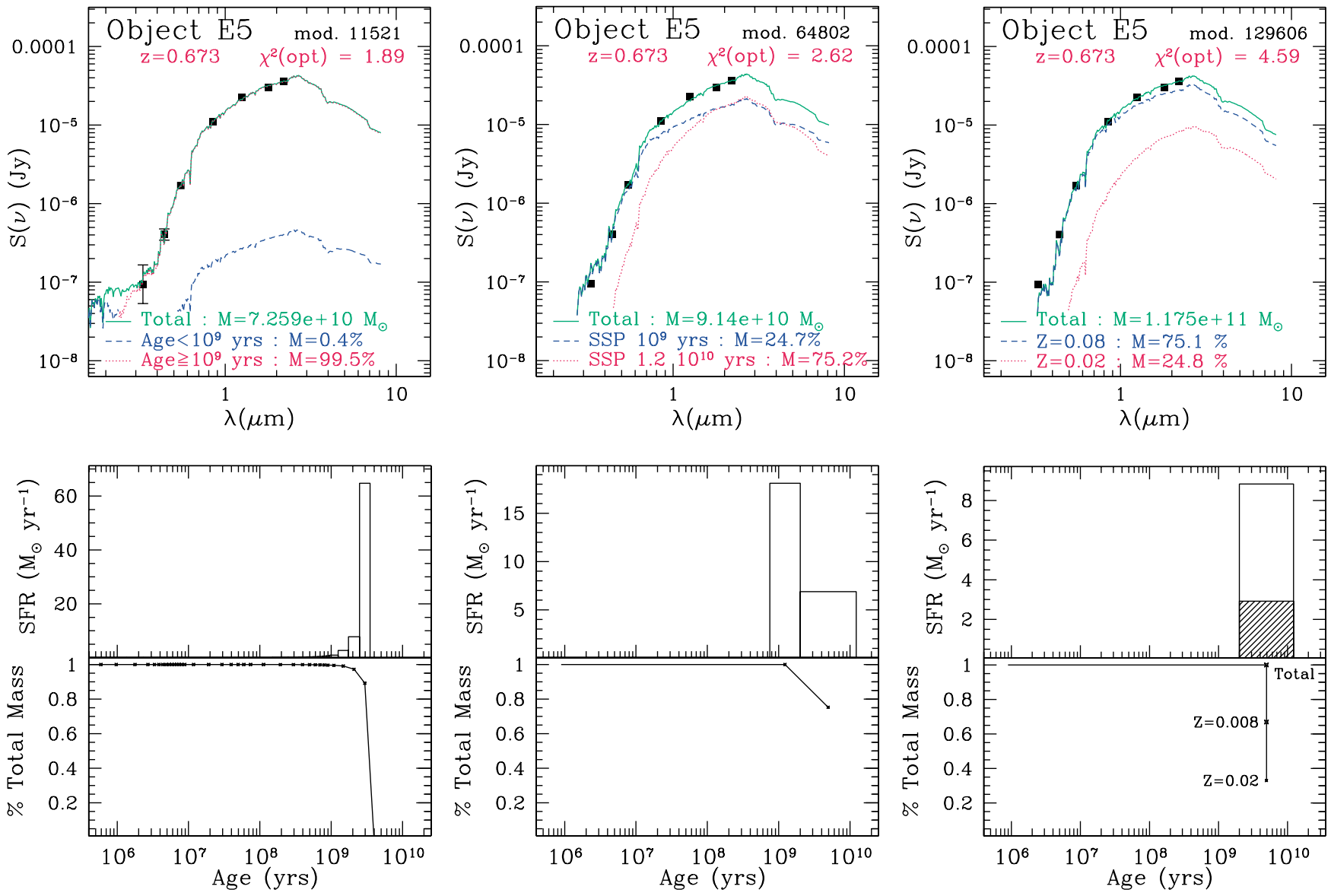

Fig. 3. Comparison of three different fits of the SED of source E5 in the ellipticals HDFS sample by Rodighiero et al. (2001), obtained with the three different adopted methods. Left: analytic star formation history, given by a Schmidt law; center: two simple stellar populations (10 ${ }^{9}$ and $1.2 \times 10^{10} \mathrm{yrs}$ old) with solar metallicity; right: two old populations $\left(1.2 \times 10^{10} \mathrm{yrs}\right)$ with different metallicities $(Z=0.02,0.008)$. Top: the best fit models SEDs (solid line) have been split into two components: on the left into the contributions of SSPs younger than $10^{9}$ yrs (dashed line) and older (dotted line); at center into the contribution of the $10^{9}$ yrs old SSP (dashed) and of the $1.2 \times 10^{10}$ yrs old one (dotted): on the right into the contribution of the $Z=0.008 \mathrm{SSP}$ (dashed) and of the $Z=0.02$ one (dotted). Bottom: star formation history and contribution of each population to the total stellar mass assembled in the galaxy. In the bottom-right panel the two old SSPs with different metallicities have been distinguished both in the SFH plot (shaded histogram is the $Z=0.02 \mathrm{SSP}$ ) and cumulative-mass diagram. The main contributions to the $\chi^{2}$ for the central and right solutions come from the $J$ and $V$ data respectively. No extinction is plotted, since it was assumed constant for all populations (see Table 2).

stellar masses are in fair agreement with those by Rodighiero et al. (2001).

For the blue E/S0 galaxy population, the simple combination of 2 old populations cannot reproduce the observed $U$, $B$ (and sometimes $V$ ) observed fluxes, while good solutions can be found by assuming some ongoing star formation (either within the analytic model, see Fig. 4, or by adding a third young SSP in the discrete SFH).

Finally we have investigated the implications of analysing the SEDs for this class of galaxies with the most general free-form spectro-photometric tool based on the multi-SSP approach and including stronger extinction effects. The only assumption in this experiment is that we have an upper limit to the mid- and far-IR flux to constrain possible dust re-radiation (we put a limit of $70 \mu \mathrm{Jy}$ at $24 \mu \mathrm{m}$, a value easily achievable with SIRTF observations). For red ellipticals this increased freedom in the spectral analysis has no effect in modifying the stellar mass estimate, while for blue ellipticals the uncertainty range increases by typically $20 \%$, with respect to the case when we can exclude a priori important extinction e.g. based on imaging data.

\subsection{Comparison of stellar masses for moderate-z starbursts and E/SO and high-z Ly-break galaxies}

Figure 5 reports the best fit solutions for four of the Lymanbreak sample galaxies based on models including only populations younger than $10^{9} \mathrm{yrs}$. The right-hand panels, top to bottom, of the figure illustrate the consequence of introducing an increasing fraction of 1-2 Gyr-old SSP in the spectrum of source Ly1. The overall fit to the observed opticalNIR data and $\chi^{2}$ values do not change, but the synthetic SED longwards of the $K$-band is significantly modified. As a consequence, without constraints on the rest-frame near-IR spectrum 

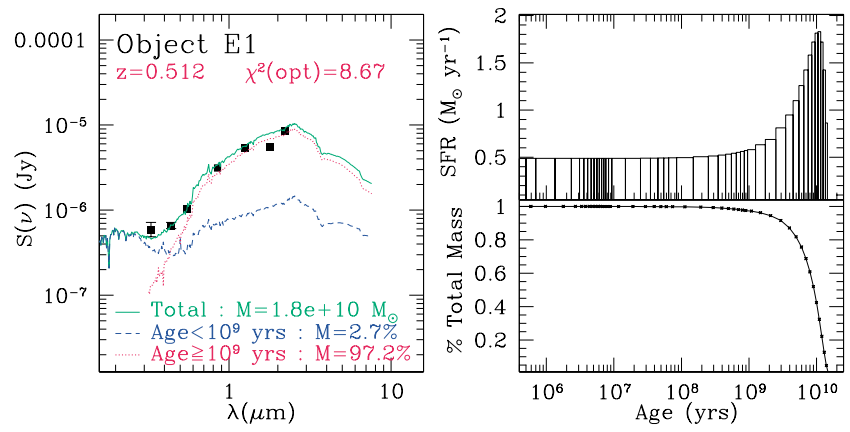

(a) E1: $z=0.512$, best fit $M=1.8 \times 10^{10} M_{\odot}$.
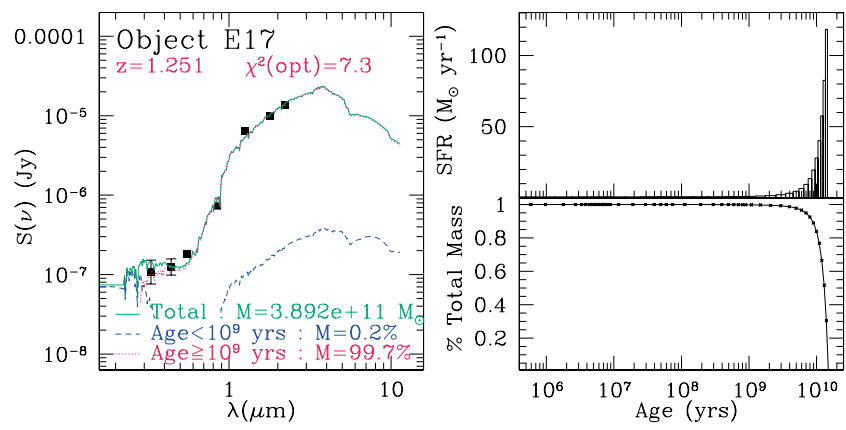

(c) E17: $z=1.25$, best fit $M=3.8910^{11} M_{\odot}$.
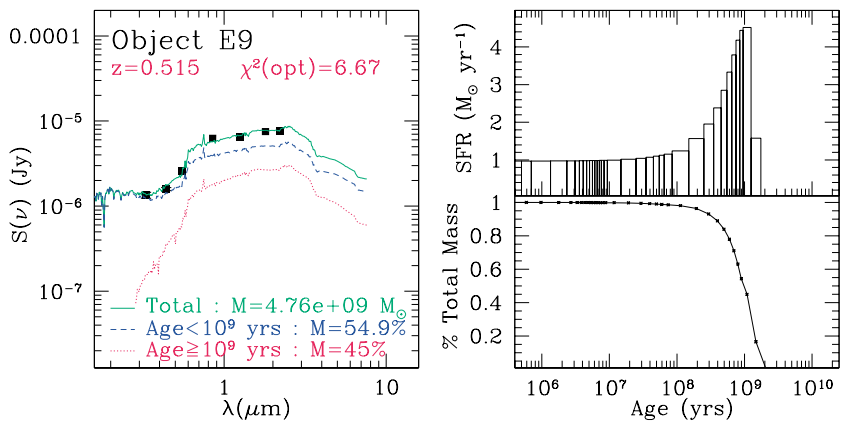

(b) E9: $z=0.515$, best fit $M=5.010^{9} M_{\odot}$.
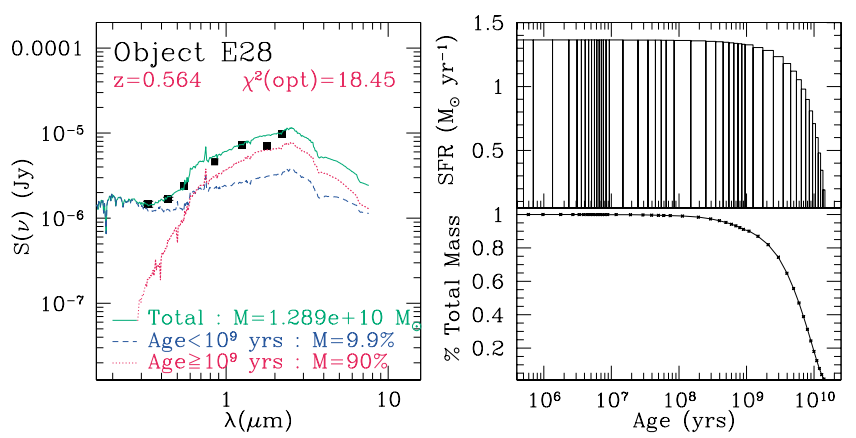

(d) E28: $z=0.564$, best fit $M=1.310^{10} M_{\odot}$.

Fig. 4. Best-fits spectral solutions for representative elliptical galaxies, based on the Schimdt star formation history model (see Sect. 3.3). See also Figs. 1 and 3.

where the bulk of the emission by low-mass stars is expected, the uncertainties in the photometric mass estimates are very large, typically 1 order of magnitude (see numerical details in Table 3).

Figure 6 plots the estimated baryonic masses for our three galaxy samples as a function of redshift. On one side, this provides evidence that the IR-selected starbursts and the $K$-band selected ellipticals and S0 galaxies seem to be comparably massive systems. Note however that the small sampled volume may imply important cosmic variance effects (e.g. some structures are evident in the HDF South samples around $z=0.5-0.6$, including galaxies of huge stellar masses).

Figure 6 shows that, in spite of the large mass uncertainties, our small illustrative sample of Lyman-break galaxies seem to correspond to a galaxy population significantly less massive ( $M<$ a few $10^{10} M_{\odot}$ ) then those observed at lower redshifts (for which typically $M>$ several $10^{10} M_{\odot}$, except for $z<0.7$ where low-mass galaxies become detectable) over comparable sky areas. Similar results were found by Papovich et al. (2001) on the sample of HDFN faint Lyman-break galaxies, with a median stellar mass of $M \sim 6 \times 10^{9} M_{\odot}$ and by Shapley et al. (2001) on brighter Ly-break galaxies (with a slightly higher median mass). Although not conclusive, due to the lack of statistics and completeness, these results indicate substantial stellar build-up to happen at $z \sim 1$ to 2 in the field population. This seems consistent with the steady increase of galaxy's $M / L$ from $z=3$ to $z=0$ and the comoving stellar mass density increasing by a factor of $\sim 10$ between $z=2$ and $z=1$ claimed by Dickinson et al. (2003).

\section{Perspectives for the SIRTF mission}

The newly operative NASA's Space InfraRed Telescope Facility (SIRTF, now Spitzer) includes a sensitive camera (IRAC) to observe high- $z$ galaxies in complementary longwavelength channels between 3 and $10 \mu \mathrm{m}$, hence providing us with a unique opportunity for the analysis of stellar masses at intermediate and high (0.5-3) redshifts, by sampling the critical restframe near-IR domain.

We have simulated SIRTF/IRAC observations based on the best fit models of some sources in the selected starburst and Lyman-break galaxy samples, to attempt to quantify how such observations will improve the mass estimates.

\subsection{Constraining the mass of active starbursts at $z=0.5-1.5$}

In the case of the IR-selected starbursts, we have found uncertainties in the stellar mass estimate based on the optical/NIR+LW3 SED of typically factors of 3-4. SIRTF/IRAC, observing at 3.6, 4.5, 5.8 and $8.0 \mu \mathrm{m}$, will significantly improve upon this. Figure 7 illustrates this with an example. The 

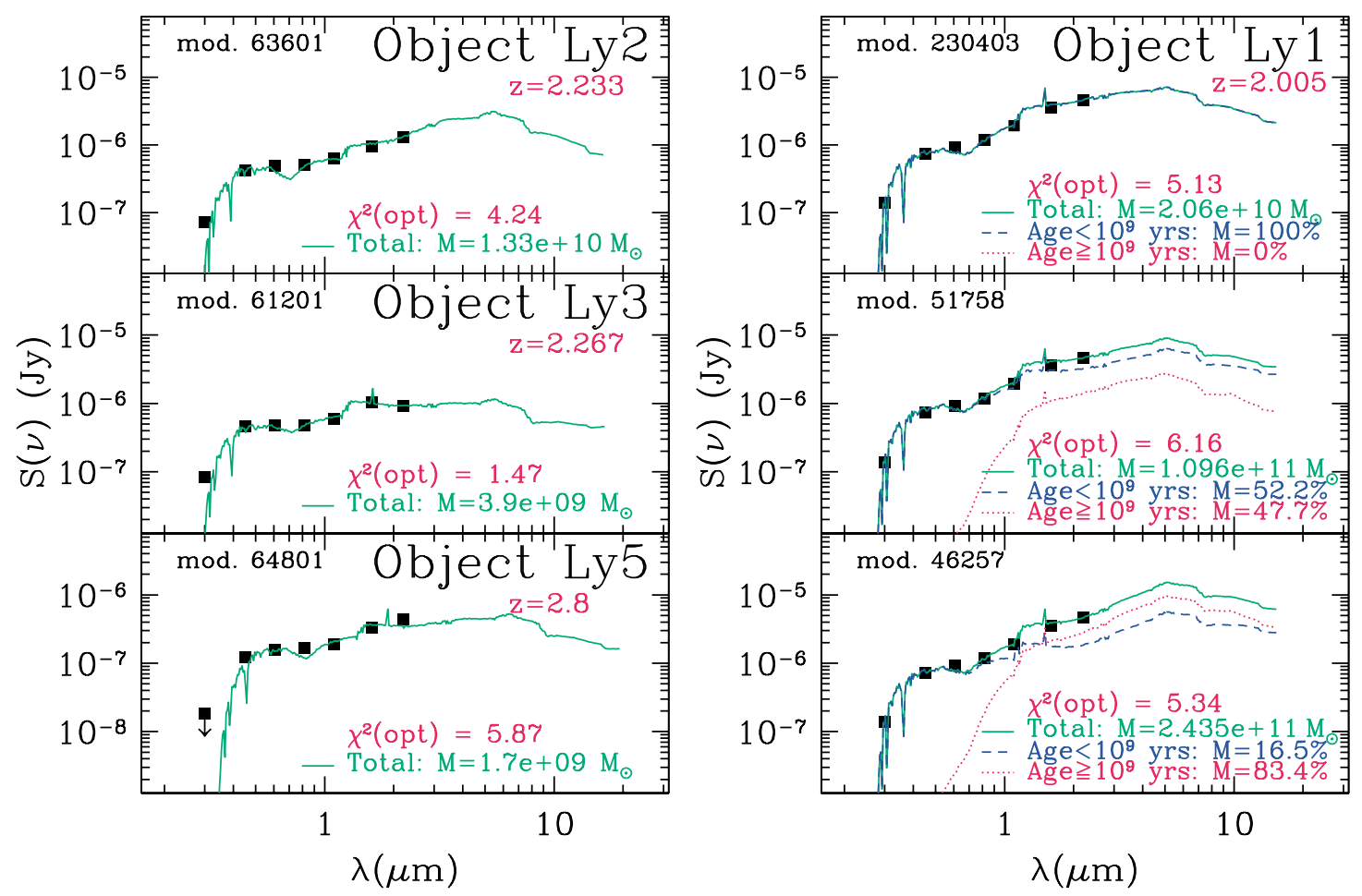

Fig. 5. SEDs fits of some Lyman-break galaxies. Left: best fit solutions for sources Ly2, Ly3 and Ly5, as obtained involving only young SSPs. Right: consequences of introducing the $\geq 10^{9} \mathrm{yrs}$ old populations on the fit of source Ly1. The relative contribution of old stars to the total assembly of stellar mass increases from top to bottom: the fit accuracy does not change, but in the restframe 1-2 $\mu$ m domain the model becomes significatively steeper (see also right panel of Fig. 7 and text for details).

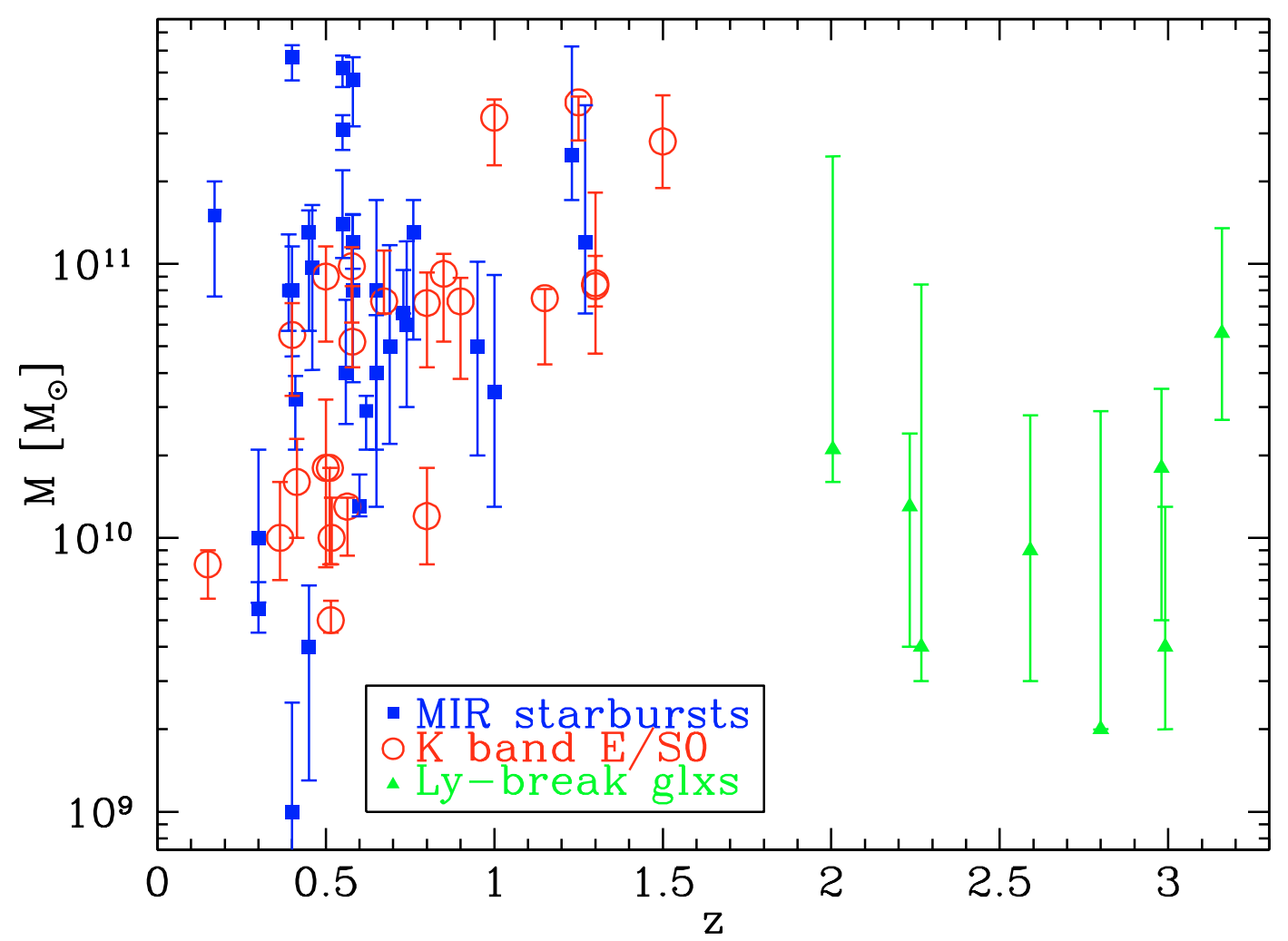

Fig. 6. Comparison of stellar masses between mid-IR selected starburst (filled squares), $K$-band selected E/S0 (open circles) and Ly-break galaxies (triangles), as a function of redshift. With the exception of very massive MIR sources belonging to the HDFS well-known group at $z \simeq 0.6$, the stellar masses we find for starbursts and $\mathrm{E} / \mathrm{S} 0$ are comparable; the small sample of Ly-break galaxies seems to consist of less massive objects than those observed at lower redshift. 

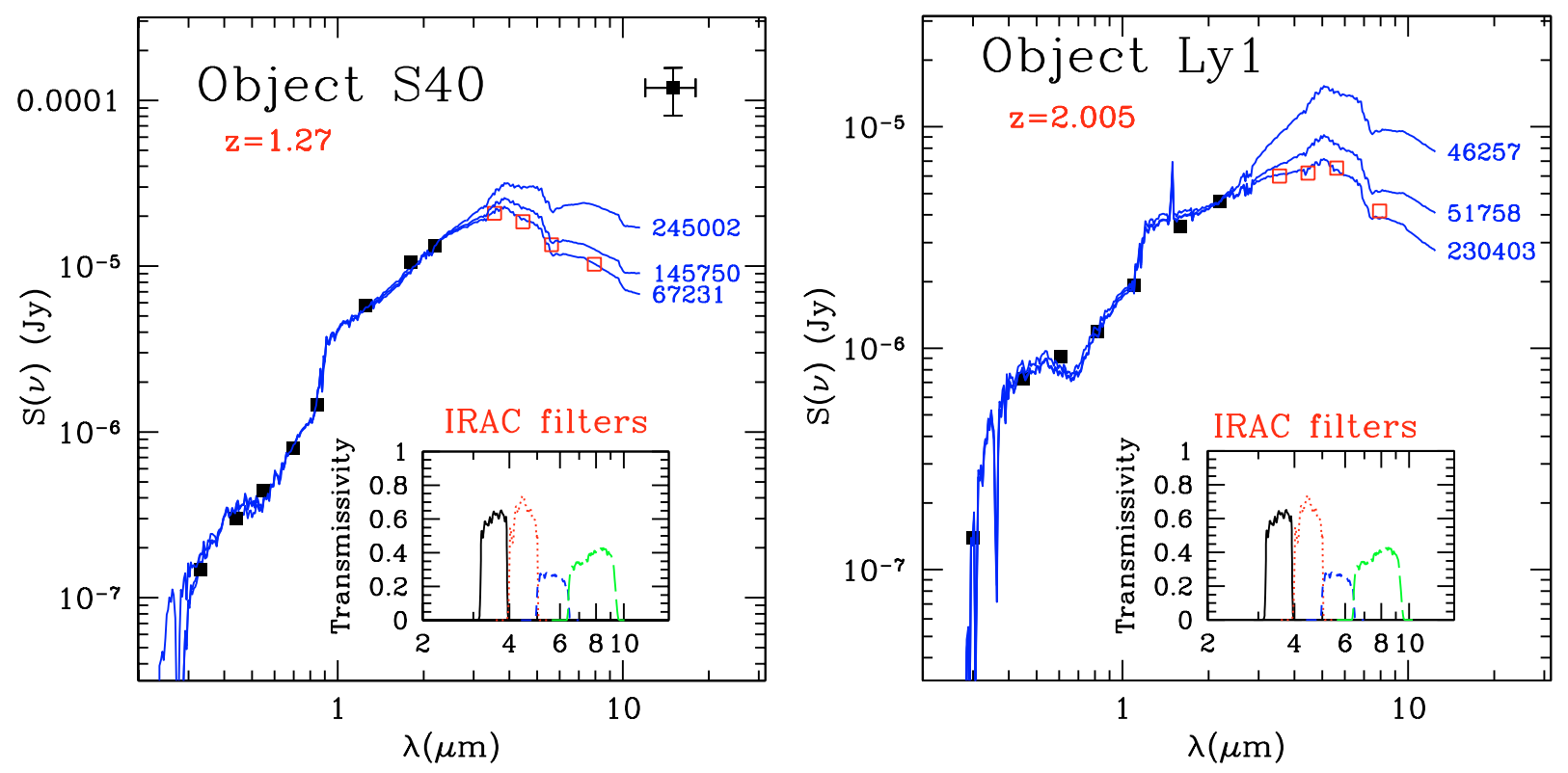

Fig. 7. Simulations of SIRTF/IRAC observations to constrain the estimate of stellar masses in starbursts and Lyman-break galaxies. Left panel: three different solutions for ISO/LW3 source S40: all three fits reproduce comparatively well both the optical data and the LW3 observed flux (even if not displayed for plotting reasons). SIRTF synthetic data (open squares) have been obtained simulating an IRAC observation of the best fit model. Right panel: the three different fits to optical-NIR data of source Ly1 already discussed in Fig. 5. IRAC synthetic fluxes have been computed for the best fit solution, which is the youngest and least massive among the three considered. In both panels the three models differ in their star formation history: the reddest are dominated by old populations more than the others. Small boxes show the transmissivity curves of the four IRAC filters; the numbers of the models refer to those appearing in Figs. 1 and 5.

open squares are the expected fluxes for galaxy S40 in the SIRTF/IRAC bands, assuming our best-fit spectral solution. Some other solutions, characterized by different SFHs, are also plotted. The case of S40 is representative of the high redshift population, while for intermediate $z$ objects (e.g. S53 or S55, $z \sim 0.5-0.7)$ the improvement may be less significant.

We have simulated a SIRTF/IRAC observation of twelve randomly-selected sources in the LW3 dusty starburst sample. We have convolved the best-fit optical/NIR SED with the 4 IRAC band transmission curves. Then we have run our code of spectral synthesis on the 12 objects, assuming IRAC simulated fluxes with a $10 \%$ photometric errors. As a result, the estimated stellar-mass range is expected to reduce to a typical factor of $\sim 2$, as reported in the last column of Table 1 .

\subsection{Detecting NIR restframe emission of $z=2-3$ Lyman-break galaxies}

Lying at redshifts $z>2$, the Lyman-break galaxies in our sample will benefit by IRAC observations much more than intermediate and low $z$ galaxies. In this redshift range, IRAC $3-8 \mu \mathrm{m}$ data will sample the restframe near-IR emission of galaxies, hence observing the light emitted by low-mass stars dominating the galaxy stellar content.

We simulated IRAC observations based on our best fit solutions. Then we have applied our spectral synthesis code using 11 datapoints, namely the already available $U B V I J H K$ photometric data and the synthetic IRAC 3.6, 4.5, 5.8 and $8.0 \mu \mathrm{m}$ fluxes. Figure 7 (right-hand panel) shows how IRAC observations will constrain the three solutions shown in the right panel of Fig. 5.

The results of these simulations are summarized in the last column of Table 3: the mass uncertainty very significantly reduces to a factor of $\sim 2-3$.

\section{Summary}

We have presented a new spectrophotometric synthesis code for the integrated light of distant and high-redshift galaxies. This tool is aimed, in perspective, at investigating various fundamental physical parameters, like the age of the dominant stellar populations, extinction, SF history, ongoing star-formation rate, etc. We have concentrated in this paper to study in particular the ability of the code to constrain the stellar mass from SEDs fitting. The tool has been tested on a sample of luminous infrared starbursts detected by ISO in the Hubble Deep Field South at $15 \mu \mathrm{m}$ (Franceschini et al. 2003), on a HDFS $K$-band selected sample of ellipticals and S0s (Rodighiero et al. 2001), and on a set of the Lyman-break galaxies in the HDF South from Papovich et al. (2001).

We have fitted the optical-NIR SEDs (from $U$ to $K$ bands) of the mid-IR starbursts by assuming a discrete star formation history between $1.2 \times 10^{10}$ years ago and today, by means of the combination of up to 10 simple stellar populations. Each SSP was weighted by different SFR's and absorbed by different amounts of dust. For a best exploitation of the available constraints we also included in the analysis the $15 \mu \mathrm{m}$ ISO flux, to assess the contribution of extinguished young stars to the emitted spectrum. Based on these data, our estimated mass uncertainties range from a factor $\sim 2$ to occasionally a factor of 5 . 
Table 3. Results of the spectral synthesis analysis of the Lyman-break galaxies. Objects are identified by numbers adopted in this work, by Papovich and by Williams (first three columns). For each source the table reports redshift, total number of models analyzed, effective number of SSPs involved in the best fit, stellar mass and $\chi^{2}$ of best fit and $2 \sigma$ mass range due to degeneracies. Last column summarizes the results of our simulated SIRTF/IRAC observations of ly-break sources: IRAC will sample the restframe near-IR light at $z \sim 2-3$, therefore significantly reducing the uncertainty on the stellar mass estimate (see text for details).

\begin{tabular}{|c|c|c|c|c|c|c|c|c|c|}
\hline $\begin{array}{l}\text { Obj } \\
\#\end{array}$ & $\begin{array}{l}\text { ID Pap. } \\
\text { (1) }\end{array}$ & $\begin{array}{l}\text { ID Will. } \\
(2)\end{array}$ & $\begin{array}{c}z \\
(3)\end{array}$ & $\begin{array}{l}N_{\text {tot }} \\
\text { mdls. }\end{array}$ & $\begin{array}{c}N_{\mathrm{SSP}} \\
\text { b.f. }\end{array}$ & $\begin{array}{l}\chi_{\mathrm{opt}}^{2} \\
\text { b.f. }\end{array}$ & $\begin{array}{l}\text { Mass (b.f.) } \\
{\left[10^{11} M_{\odot}\right]}\end{array}$ & $\begin{array}{c}\Delta M \\
{\left[10^{11} M_{\odot}\right]}\end{array}$ & $\begin{array}{c}\Delta M_{\mathrm{SIRTF}} \\
{\left[10^{11} M_{\odot}\right]}\end{array}$ \\
\hline Ly1 & 110 & $2-449.0$ & 2.005 & 230403 & 3 & 5.13 & 0.21 & $0.16-2.47$ & $0.27-0.55$ \\
\hline Ly2 & 503 & $2-903.0$ & 2.233 & 195602 & 3 & 4.24 & 0.13 & $0.04-0.24$ & $0.09-0.19$ \\
\hline Ly3 & 67 & $2-82.1$ & 2.267 & 195587 & 3 & 1.47 & 0.04 & $0.03-0.84$ & $0.04-0.09$ \\
\hline Ly4 & 804 & 4-639.0 & 2.591 & 212778 & 2 & 10.99 & 0.09 & $0.03-0.28$ & $0.03-0.09$ \\
\hline Ly5 & 1352 & $4-497.0$ & 2.800 & 199203 & 3 & 5.87 & 0.02 & $0.02-0.29$ & $0.02-0.06$ \\
\hline Ly6 & 1541 & $4-363.0$ & 2.980 & 217529 & 3 & 9.02 & 0.18 & $0.05-0.35$ & $0.06-0.20$ \\
\hline Ly7 & 661 & $2-643.0$ & 2.991 & 264435 & 2 & 7.54 & 0.04 & $0.02-0.13$ & $0.03-0.06$ \\
\hline Ly8 & 273 & $2-76.11$ & 3.160 & 278399 & 2 & 2.29 & 0.56 & $0.27-1.35$ & $0.38-0.81$ \\
\hline
\end{tabular}

(1): ID number in Papovich et al. (2001);

(2): ID number in Williams et al. (1996);

(3): Spectroscopic redshifts by Steidel et al. (1996) and Lowental et al. (1997).

In such dust-obscured starburst galaxies, substantial uncertainty in the mass estimate comes from the still possible existence of strongly extinguished young stars with low $M / L$ ratios, contributing to the far-IR flux but undetectable in the rest-frame optical.

We have also analysed a sample of morphologicallyselected E/S0 galaxies, at $z<1.5$. Their SEDs have been reproduced as the combination of stellar populations with solar metallicities, or by combining SSPs of different metallicities $(Z=0.02$ and $Z=0.008)$. Additional solutions have been sought with a parameterized continuous sequence of stellar ages. The SEDs of spheroidal galaxies with red colors are well fitted by combining intermediate and old populations only, while for ellipticals with relatively blue colors (the majority in our complete sample) acceptable solutions are found only by assuming some recent or even ongoing star formation. The three different methods provide results well consistent with each other.

For Lyman-break galaxies the available rest-frame opticalUV data are much less constraining. The consequences of assuming relatively old $\left(\sim 10^{9} \mathrm{yrs}\right)$ stellar populations in these systems have been discussed and it has been found that the lack of long-wavelength data imply uncertainties in the mass estimate of up to a factor of $\simeq 10$. In spite of these large uncertainties, there are indications that typical Lyman-break galaxies correspond to a galaxy population significantly less massive then those observed at lower redshifts, possibly indicating substantial stellar build-up to ocurring at $z \sim 1$ to 2 in the field galaxy population.

The situation is expected to significantly improve, particularly for higher- $z$ galaxies, with the forthcoming SIRTF/IRAC observations. Our simulations show that such an extension of the wavelengths dynamic range will reduce the mass uncertainties to factors of 2-3 for various classes of galaxies up to $z \sim 3$. This promises to be good enough for statistically reliable determinations of the galaxy evolutionary mass functions.

Acknowledgements. We wish to thank M. Rowan-Robinson for useful and interesting discussions on SEDs fitting and degeneracies. S.B. work was supported by ASI research grant No. I/R/062/02. We are grateful to the anonymous referee for his very useful comments.

\section{References}

Berta, S., Fritz, J., Franceschini, A., Bressan, A., \& Pernechele, C. 2003, A\&A, 403, 119

Bertin, E., \& Arnouts, S. 1996, A\&AS, 117, 393

Cardelli, J. A., Clayton, G. C., \& Mathis, J. S. 1989, ApJ, 345, 245

Carroll, S. M., Press, W. H., \& Turner, E. L. 1992, ARA\&A, 30, 499

Corana, et al. 1987, Minimizing Multimodal Functions of Continuous Variables with the Simulated Annealing Algorithm, in ACM Transactions on Mathematical Software, 13, No. 3, 262

Da Costa, L., Nonino, M., Rengelink, R., et al. 1998, [arXiv: astro-ph/9812105]

Dickinson, M., Papovich, C., Ferguson, H. C., \& Budavari, T. 2003, ApJ, 587, 25

Elbaz, D., Cesarsky, C., Chanial, P., et al. 2002, A\&A, 384, 848

Ferland, G. J. 1996, Hazy, a Brief Introduction to CLOUDY, in University of Kentucky, Departmnt of Physics and Astronomy Internal Report

Franceschini, A., Silva, L., Fasano, G., et al. 1998, ApJ, 506, 600

Franceschini, A., Lonsdale, C., et al. 2003, in The Mass of Galaxies at Low and High Redshift. Proceedings of the ESO Workshop held in Venice, Italy, 24-26 October 2001, 338

Franceschini, A., Berta, S., Rigopoulou, D., et al. 2003, A\&A, 403, 501

Kauffman, G., \& Charlot, S. 1998, MNRAS, 297, L23 
Kennicutt, R. C. 1992, ApJ, 388, 310

Kurucz, R. 1993, ATLAS9 Stellar Atmosphere Programs and 2 km/s grid, Kurucz CD-ROM No. 13, Cambridge, Mass.: Smithsonian Astrophysical Observatory

Ingber, L. 1989, Mathematical Computer Modelling, 12, 967

Ingber, L. 2001, Adaptive Simulated Annealing (ASA) and Path-Integral (PATHINT) Algorithms: Generic Tools for Complex Systems, URL: http://www.ingber.com/asa01_ lecture.pdf

Lancon, A., Mouhcine, M., Fioc, M., \& Silva, D. 1999, A\&A, 344, L21

Lowental, J. D., Koo, D. C., Guzman, R., et al. 1997, ApJ, 481, 673

Oliver, S., Mann, R. G., Carballo, R., et al. 2002, MNRAS, 332, 536

Origlia, L., \& Oliva, E. 2000, A\&A, 357, 61

Papovich, C., Dickinson, M., \& Ferguson, H. C. 2001, ApJ, 559, 620

Pickles, A. J. 1998, PASP, 110, 863

Pignatelli, E., \& Fasano, G. 1999, Ap\&SS, 269, 657
Poggianti, M. B., Bressan, A., \& Franceschini, A. 2001, ApJ, 550, 195

Rigopoulou, D., Franceschini, A., Aussel, H., et al. 2000, ApJ, 537, L85

Rodighiero, G., Franceschini, A., \& Fasano, G. 2001, MNRAS, 324, 491

Sawicki, M., \& Mallen-Ornelas, G. 2003, ApJ, accepted [arXiv: astro-ph/0305544]

Sawicki, M., \& Yee, H. K. C. 1998, ApJ, 115, 1329

Shapley, A. E., Steidel, C. C., Adelberger, K. L., et al. 2001, ApJ, 562, 95

Steidel, C. C., Giavalisco, M., Dickinson, M., \& Adelberger, K. L. 1996, AJ, 112, 352

Teplitz, H. I., Gardner, J. P., Palunas, P., et al. 1998, Am. Astron. Soc. Meet., 193, 7507

Vanzella, E., Cristiani, S., Arnouts, S., et al. 2003, A\&A, 396, 847

Williams, R. E., Blacker, B., Dickinson, M., et al. 1996, AJ, 112, 1335 